\title{
ON A PROBLEM CONNECTED WITH THE VANDERMONDE DETERMINANT
}

\author{
J. KIEFER ${ }^{1}$ AND J. WOLFOWITZ ${ }^{2}$
}

Let $m$ be a positive integer. We denote by $a=\left(a_{0}, a_{1}, \cdots, a_{m}\right)$, $x=\left(x_{0}, \cdots, x_{m-1}\right)$ vectors with real components. Define

(1)

$$
\begin{aligned}
U & =\left\{x \mid \text { all }\left|x_{i}\right| \leqq 1, x_{i} \neq x_{j}, i \neq j\right\}, \\
P^{*}(x, a) & =\left|\begin{array}{ccccc}
1 & 1 & \cdot & 1 & a_{0} \\
x_{0} & x_{1} & \cdot & x_{m-1} & a_{1} \\
x_{0}^{2} & x_{1}^{2} & \cdot & x_{m-1}^{2} & a_{2} \\
\cdot & \cdot & \cdot & \cdot & \cdot \\
\cdot & \vdots & \cdot & \cdot & \cdot \\
x_{0}^{m} & x_{1}^{m} & \cdot & x_{m-1}^{m} & a_{m}
\end{array}\right|, \\
A^{*} & =\left\{a \mid P^{*}(x, a) \neq 0 \text { for every } x \in U\right\} .
\end{aligned}
$$

This paper is devoted to the problem of characterizing $A^{*}$, a problem which is of interest in the theory of the design of statistical experiments (see [1]) and is perhaps of interest per se.

When $m=1$, it is trivial that $A^{*}=\left\{\left(a_{0}, a_{1}\right)|| a_{0}|<| a_{1} \mid\right\}$. We hereafter assume $m>1$. Define

$$
A(c)=A^{*} \cap\left\{a \mid a_{m}=c\right\} .
$$

We shall prove the following:

(I) $A(1)$ is a set $A$ described precisely below.

(II) For $c \neq 0$, we have $\left(a_{0}, a_{1}, \cdots, a_{m-1}, c\right) \in A(c)$ if and only if $\left(a_{0} / c, \cdots, a_{m-1} / c, 1\right) \in A$.

(III) $A(0)$ is empty.

Of these, (II) is obvious, and (III), which we shall prove in the paragraph after next, is only slightly less so. The problem is to describe $A$.

Let $S_{0}(x) \equiv 1$ and

Received by the editors August 24, 1964.

1 Research supported by the U. S. Office of Naval Research under contract No. Nonr 266(04) (NR 047-005).

2 Research supported by the U.S. Air Force under contract No. AR 18(600)-685, monitored by the Office of Scientific Research. 


$$
S_{j}(x) \equiv(-1)^{j} \sum x_{i_{1}} x_{i_{2}} \cdots x_{i_{j}},
$$

where the summation is with respect to $i_{1}, \cdots, i_{j}, 0 \leqq i_{1}<i_{2}<\cdots$ $<i_{j}<m$. Let

$$
P(x, a) \equiv \sum_{j=0}^{m} a_{m-j} S_{j}(x) .
$$

Expanding the determinant $P^{*}$ of (1) in minors of the last column shows that

$$
P^{*}(x, a)=P(x, a) K(x),
$$

where the polynomial $K$ is the Vandermonde determinant which is the minor of $a_{m}$ in the determinant $P^{*}$ of (1); thus, $K$ is never zero on $U$. Hence in the definition of $A^{*}$ we may replace $P^{*}(x, a)$ by $P(x, a)$.

We shall now prove that $A(0)$ is empty. Let $a$ be any $(m+1)$ vector with $a_{m}=0$. If all $a_{j}=0$, clearly $a \notin A^{*}$. Suppose then that $j_{0}$ is the smallest integer for which $a_{m-j_{0}} \neq 0,0<j_{0} \leqq m$. We shall find two points $x^{\prime}$ and $x^{\prime \prime}$ in the convex subset $T$ of $U$ where $-1 \leqq x_{0}$ $<x_{1}<\cdots<x_{m-1} \leqq 1$ such that

$$
(-1)^{j_{0}} a_{j_{0}} P\left(x^{\prime}, a\right)<0<(-1)^{j_{0}} a_{j_{0}} P\left(x^{\prime \prime}, a\right),
$$

from which it follows that $P(x, a)=0$ for some $x$ in $T$; it follows that $A(0)$ is empty. Let $x^{\prime \prime}=(\epsilon, 2 \epsilon, \cdots, m \epsilon)$ with $0<\epsilon<1$. Each term in the sum in (2) is then positive, and thus $(-1)^{j_{0}} S_{j_{0}}\left(x^{\prime \prime}\right)>\epsilon^{j_{0}}$, while $S_{j}\left(x^{\prime \prime}\right)=o\left(\epsilon^{j_{0}}\right)$ as $\epsilon \rightarrow 0$ if $j>j_{0}$. Hence, for $\epsilon$ sufficiently small, the second half of (4) follows from (3). The first half of (4) follows similarly if $j_{0}$ is odd upon taking $x^{\prime}=(-m \epsilon,-(m-1) \epsilon, \cdots,-\epsilon)$, and if $j_{0}$ is even upon taking $x^{\prime}=(-(m-1) \epsilon,-(m-2) \epsilon, \cdots,-\epsilon, 1)$.

Let

$$
\begin{aligned}
Q_{h}(x) \equiv \sum\left(1-x_{i_{1}}\right)\left(1-x_{i_{2}}\right) & \cdots\left(1-x_{i_{h}}\right)\left(1+x_{i_{h+1}}\right) \cdots \\
& \cdots\left(1+x_{i_{m}}\right) /\left(\begin{array}{c}
m \\
h
\end{array}\right), \quad 0 \leqq h \leqq m,
\end{aligned}
$$

where the summation is over the $\left(\begin{array}{c}m \\ m\end{array}\right)$ choices of the disjoint sets $\left(i_{1}, i_{2}, \cdots, i_{h}\right)$ and $\left(i_{h+1}, \cdots, i_{m}\right)$ of distinct integers between 0 and $m-1$, inclusive. Define the points

$$
a^{(h)}=\left(a_{0}^{(h)}, \cdots, a_{m}^{(h)}\right), \quad 0 \leqq h \leqq m,
$$

by 


$$
Q_{h}(x)=\sum_{j=0}^{m} a_{m-j}^{(h)} S_{j}(x) .
$$

We note that $a_{m}^{(n)}=1$ for all $h$, and that

$$
\begin{aligned}
& a^{(0)}=\left((-1)^{m},(-1)^{m-1}, \cdots,(-1)^{0}\right), \\
& a^{(m)}=(1,1, \cdots, 1) .
\end{aligned}
$$

We will now prove:

THEOREM. For $m>1, A$ is the closed $m$-simplex with extreme points $a^{(0)}, \cdots, a^{(m)}$, minus the (closed) edge which connects $a^{(0)}$ and $a^{(m)}$.

Let $\bar{U}$ be the closure of $U$. If $x$ is a vertex of $\vec{U}$ with $r$ coordinates -1 and $(m-r)$ coordinates +1 , then

$$
\begin{aligned}
Q_{h}(x) & =2^{m} /\left(\begin{array}{l}
m \\
h
\end{array}\right) & \text { if } h=r, \\
& =0 & \text { if } h \neq r .
\end{aligned}
$$

Hence the $Q_{h}$ are linearly independent functions. Moreover, each $Q_{h}(x) \geqq 0$ on $U$. If $h \neq 0$ or $m, Q_{h}(x)>0$ on $U$, since at most one $x_{i}$ can take the value +1 and at most one $x_{i}$ can take the value -1 on $U$.

According to (3), $P(x, a)$ is linear in $a$. According to (7), $P\left(x, a^{(h)}\right)$ $=Q_{h}(x)$. Combining these facts, if $\alpha_{0}, \cdots, \alpha_{m}$ are real numbers,

$$
P\left(x, \sum_{0}^{m} \alpha_{h} a^{(h)}\right)=\sum_{0}^{m} \alpha_{h} Q_{h}(x) .
$$

Since the $Q_{h}$ are linearly independent, it follows from (7) that the vectors $a^{(i)}, 0 \leqq i \leqq m$, are linearly independent. Hence, $a^{(0)}, \cdots, a^{(m)}$ lie in the hyperplane $a_{m}=1$, but in no other hyperplane. Hence, any point $a$ in the hyperplane $a_{m}=1$ can be written in a unique way as

$$
a=\sum_{0}^{m} \alpha_{h} a^{(h)}
$$

where $\sum \alpha_{h}=1$. Suppose now that $\alpha_{h} \geqq 0$ for all $h$, and $\alpha_{h}>0$ for some $h \neq 0$ or $m$. Then, from (8) and the paragraph below the statement of the theorem, we have $P(x, a)>0$ for all $x$ in $U$. From this it follows that $A$ is at least as large as stated in the theorem. It remains to prove that it is no larger.

If $\alpha_{0}+\alpha_{m}=1$ and all other $\alpha_{h}=0$, we have $P(x, a)=0$ for any $x$ in $U$ such that $x_{0}=1, x_{1}=-1$. Hence the closed segment $\left[a^{(0)}, a^{(m)}\right]$ is not in $A$. 
Suppose now that $\alpha_{i_{0}}<0$. If 0 is the origin then of course $P(0, a)=1$. It follows from the paragraph below the statement of the theorem that there is an $x^{*}$ in $U$ such that (i) the half-open segment $\left(0, x^{*}\right]$ is in $U$, (ii) $Q_{i_{0}}\left(x^{*}\right)>\frac{1}{2}$, and (iii) for $j \neq i_{0}, Q_{j}\left(x^{*}\right)<\left|\alpha_{i_{0}}\right| / 2 \sum\left|\alpha_{h}\right|$. From (8) we have $P\left(x^{*}, a\right)<0$. Hence, for some point $y \in\left(0, x^{*}\right)$ (so that $y \in U$ ) we must have $P(y, a)=0$. Hence $a$ cannot be in $A$. The proof of the theorem is complete.

We remark that the points $a$ of the form $\left(b^{m}, b^{m-1}, \cdots, b^{1}, 1\right)$ with $|b|<1$ are easily verified to be in $A$; this is of interest in applications.

\section{REFERENCE}

1. J. Kiefer and J. Wolfowitz, On a theorem of Hoel and Levine on extrapolation designs, Ann. Math. Statist. (to appear).

Cornell University 